\title{
Commentary: No one drug does only one thing in only one setting
}

\author{
Jakob Vinten-Johansen, PhD
}

\author{
From the Department of Surgery (Cardiothoracic Surgery), Emory University, Atlanta, Ga. \\ Disclosures: Author has nothing to disclose with regard to commercial support. \\ Received for publication Feb 18, 2019; accepted for publication Feb 18, 2019; available ahead of print March 23, \\ 2019. \\ Address for reprints: Jakob Vinten-Johansen, PhD, 159 Montauk Ct, Clemmons, NC 27012 (E-mail: jvinten@ \\ emory.edu). \\ J Thorac Cardiovasc Surg 2019;158:299-300 \\ $0022-5223 / \$ 36.00$ \\ Copyright (C) 2019 by The American Association for Thoracic Surgery \\ https://doi.org/10.1016/j.jtcvs.2019.02.081
}

A well-worn cliché states that no one drug does only one thing. This can be an advantage when the drug's actions are applicable to other pathologies. On the basis of reports of anti-inflammatory and antioxidant actions of the antifibrotic agent pirfenidone, Saito and colleagues ${ }^{1}$ conducted a proof-of-concept exploration, reported in this issue of the Journal, of the efficacy of pirfenidone in attenuating lung ischemia-reperfusion injury - a different setting that shares some pathophysiology with idiopathic pulmonary fibrosis. With a rodent model of acute single-lung ischemia-reperfusion, Saito and colleagues ${ }^{1}$ report that oral pirfenidone pretreatment (1) attenuated loss of compliance, (2) improved arterial oxygenation, (3) reduced perivascular edema and the presence of neutrophils and apoptotic cells, and (4) decreased expression of proinflammatory mediator messenger RNA after ischemiareperfusion relative to the untreated group. The conclusion that pirfenidone inhibits experimental acute pulmonary ischemia-reperfusion injury is supported by the data.

The study by Saito and colleagues ${ }^{1}$ has several strengths. It is innovative, albeit observational. The study was not designed to determine dose-response characteristics, nor to determine mechanisms of action or molecular targets. The end points were relevant to the pathophysiology of pulmonary ischemia-reperfusion injury. The research design appropriately included time-matched sham and control (untreated) ischemia-reperfusion groups. All groups received the vehicle used to dissolve pirfenidone, thereby avoiding any confounding between-group effects attributable to the vehicle itself. Notably, Saito and colleagues ${ }^{1}$ measured blood and lung tissue concentration of pirfenidone, demonstrating that oral pirfenidone was systemically present. Key aspects of histologic and messenger RNA data were quantified, to which statistics were applied.

The study by Saito and colleagues ${ }^{1}$ also has several weaknesses. Although the rat model is inexpensive and less labor-intensive than larger animal models, rodent inflammatory biology has notable differences from that of other species, which may question rodent data's

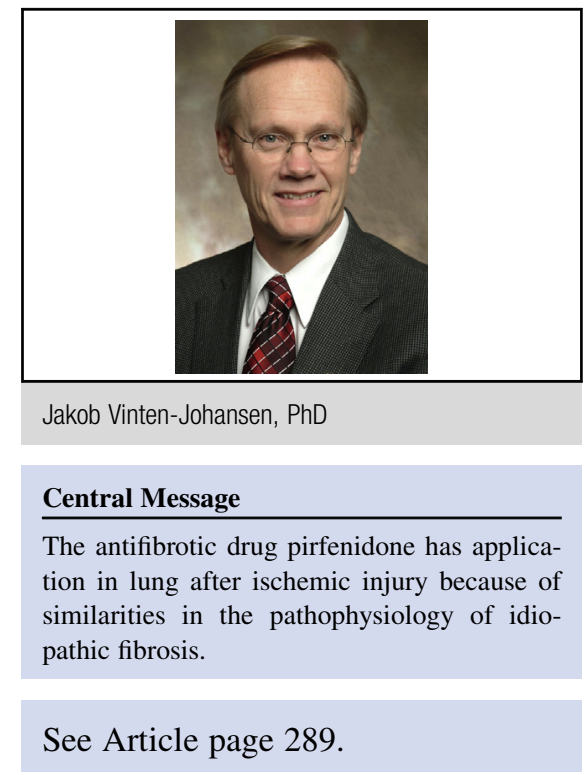

applicability to those species. The messenger RNA data could have been further strengthened by measuring respective protein levels in lung tissue. Although Saito and colleagues ${ }^{1}$ frequently state that the in vivo ischemiareperfusion model simulates lung transplantation, flushing and storage solutions with various compositions and temperatures used in transplantation may present a different pathobiology than warm ischemia-reperfusion. Furthermore, it is not clear whether, in practice, efficacy would require administration of pirfenidone to both donor and recipient of the lung transplant.

There are many next steps required to develop pirfenidone for use in lung ischemia-reperfusion injury, least of which is testing in large animal survival studies. But this study of Saito and colleagues ${ }^{1}$ is a necessary first step. On the basis of inhibition of proinflammatory mediator and reactive oxygen species $^{2}$ generation by pirfenidone, and the reported direct effects on promoting contractile function, ${ }^{3}$ pirfenidone may have exciting potential in the heart. Reports suggest pirfenidone is effective in reducing infarct size and fibrosis in myocardial infarction. ${ }^{4}$ Pirfenidone may also be effective as an adjunct to cardioprotective strategies designed to attenuate postcardioplegia pathology. Likewise, pirfenidone may be useful in cardiac transplantation when added to storage solutions and administered after transplant. Pirfenidone may also be applicable in other organ systems that share inflammatory and oxidant mechanisms. The study by Saito and 
colleagues ${ }^{1}$ is a first step in seeking to broaden applications for pirfenidone to lung protection and beyond.

\section{References}

1. Saito M, Chen-Yoshikawa TF, Suetsugu K, Okabe R, Takahagi A, Masuda S, et al. Pirfenidone alleviates lung ischemia-reperfusion injury in a rat model. J Thorac Cardiovasc Surg. 2019;158:289-96.
2. Schaefer CJ, Ruhrmund DW, Pan L, Seiwert SD, Kossen K. Antifibrotic activities of pirfenidone in animal models. Eur Respir Rev. 2011;20:85-97.

3. Monsalvo-Villegas A, Osornio-Garduño DS, Avila G. Long-term regulation of excitation-contraction coupling and oxidative stress in cardiac myocytes by pirfenidone. Front Physiol. 2018;9:1801.

4. Nguyen DT, Ding C, Wilson E, Marcus GM, Olgin JE. Pirfenidone mitigates left ventricular fibrosis and dysfunction after myocardial infarction and reduces arrhythmias. Heart Rhythm. 2010;7:1438-45. 